\title{
The Surgical Management of Retrosternal (Intrathoracic) Goiter
}

Malcolm H Wheeler

\section{ABSTRACT}

Retrosternal (intrathoracic) goiter has a prevalence of 4 to $20 \%$ of patients undergoing thyroidectomy for multinodular goiter. Although it is a condition affecting the older patient with a peak incidence in the sixth decade, the majority should be treated surgically. With few exceptions the operation can be safely accomplished through a cervical approach, only a small minority (approximately $2 \%$ ) needing a combined cervical/mediastinal procedure. The surgical steps for the routine case are described and also various technical maneuvers required to facilitate delivery and excision of the difficult large goiter.

Keywords: Thyroid, Retrosternal goiter, Surgical management.

How to cite this article: Wheeler $\mathrm{MH}$. The Surgical Management of Retrosternal (Intrathoracic) Goiter. World J Endoc Surg 2013;5(1):28-30.

Source of support: Nil

Conflict of interest: None

\section{INTRODUCTION}

The definition of an intrathoracic goiter remains somewhat confused with some including any goiter with the lower pole lying below the thoracic inlet and others reserving the definition only for those glands in which at least $50 \%$ of the thyroid is below the inlet. ${ }^{1}$ This apparent discrepancy accounts for the wide variation in quoted prevalence of the condition ranging from 4 to $20 \%$ of patients undergoing surgery for multinodular goiter. ${ }^{2}$ Mediastinal extension is more common in large multinodular and recurrent goiters with the peak incidence occurring in the sixth decade. It is not unusual for such patients to have coexisting pathology with thyroid malignancy present in 3 to $17 \%{ }^{3-5}$ These factors clearly have a major influence on the overall management strategy. Nevertheless unless there are strong medical contraindications to surgery the majority of retrosternal goiters should be treated by thyroidectomy.

Preoperative diagnosis of retrosternal extension is not always straightforward, the classical symptoms of breathlessness in an elderly patient with goiter often being attributed to respiratory disease, such as asthma or chronic bronchitis. In this regard lung function tests (peak expiratory flow, forced expiratory volume and flow loop studies) can be extremely helpful.

In 20 to $30 \%$ of cases the thyroid gland is not palpable in the neck because the thoracic retrosternal extension represents most of the gland. ${ }^{5,6}$
The combination of chest radiography with either computed tomographic (CT) scanning or magnetic resonance imaging (MRI) will provide valuable preoperative information regarding degree of intrathoracic extension, precise location of extension (anterior substernal, posterior retroesophageal), limit of extension inferiorly, tracheal compression or deviation and vascular invasion by a malignant thyroid.

Appropriate thyroid function tests determine thyroid status and a preoperative laryngoscopy is mandatory to exclude a laryngeal nerve palsy and vocal cord paralysis which may accompany a benign gland because of direct pressure or a thyroid cancer due to nerve invasion.

The majority of retrosternal goiters can be delivered and safely resected through a standard cervical thyroidectomy approach, only approximately $2 \%$ requiring a combined cervical/sternotomy procedure. ${ }^{2}$ This latter approach is most likely to be necessary with very large goiters where crosssectional imaging may show a large discrepancy in size between the cervical and thoracic components, retroesophageal extension and very deeply situated tumors with evidence of malignant invasion. But it is rarely possible to accurately predict preoperatively that a sternotomy will be required. It is therefore wise to err on the side of caution, prepare the patient for this possibility and drape the chest as well as the neck for any large retrosternal goiter.

In spite of these considerations and potential pitfalls thyroidectomy for retrosternal glands is usually straightforward and accomplished without difficulty.

Marked venous engorgement and even frank superior vena cava obstruction are well known accompaniments of large retrosternal glands and therefore the patient should be placed on the operating table in a somewhat exaggerated head-up position and the neck well extended. These measures will help to reduce venous pressure and mitigate the risk of unnecessary bleeding. As in all thyroid operations it is essential to proceed in a bloodless field so that important structures, such as the parathyroid glands and recurrent laryngeal nerves (RLN) can be identified and preserved.

In order to gain comfortable access to a gland which extends down into the chest it is necessary to place the collar incision at a level 1 to $2 \mathrm{~cm}$ higher than usual. Division of the strap muscles is not routinely required but in the case of 
a goiter with a large proportion of the gland situated in the chest there should be no hesitation in dividing these muscles on one or both sides in order to facilitate access.

The initial key steps before any attempt is made to mobilize or deliver the retrosternal component are ligation and division of the superior thyroid vessels with the usual precautions taken to identify and preserve the external branch of the superior laryngeal nerve. These essential steps impart mobility to the upper pole of the thyroid and will greatly facilitate the subsequent upward movement and delivery into the neck of the thyroid with its retrosternal prolongation.

If the middle thyroid veins can be identified at this stage they should be ligated and divided before starting to mobilize the thoracic gland.

The lateral attachments of the cervical gland are dissected free before focusing attention on the posterior aspects of the cervical thyroid, in which area it is usually possible to identify the superior parathyroid gland and the RLN. Palpation of the inferior cornu of the thyroid cartilage is a valuable landmark to identify the location and point of entry of the RLN into the larynx.

The fascial attachments and adhesions of the thyroid are freed with a finger inferiorly, especially between the gland and the posterior aspect of the manubrium. Gentle upward traction with the other hand and often some judicious sharp scissor dissection under direct vision will almost invariably permit successful delivery of the thyroid into the neck.

It is hoped that the inferior parathyroid gland can be identified close to the posterolateral aspect of the lower pole. Depending on its precise location and vascular status such a gland may require autotransplantation into the sternomastoid muscle.

The above maneuvers may not always permit delivery of a large retrosternal gland. In these circumstances confirmation that all accessible adhesions have been divided is made by again sweeping the index finger around the intrathoracic gland. Of course a thyroid extending far inferiorly is likely to have a distal pole which is beyond the reach of a finger. A sterile soup spoon can be used to advantage: It may be slipped cautiously down alongside the anterolateral aspect of the thyroid, breaking negative intrathoracic pressure, reaching further into the chest than the finger, occupying less space and, in most instances, leading to an immediate and satisfying delivery of the gland. ${ }^{1}$

A note of caution is required at this point. Excessive, enthusiastic traction must be avoided for fear of stretching and damaging the RLN even though it may already have been identified at a higher level in the neck. Indeed the surgeon must be aware that an RLN can be stretched over a large component of a retrosternal goiter and thereby displaced well away from its usual and expected position. A nerve so situated is undoubtedly at considerable risk (Fig. 1).

If none of the aforementioned measures succeed a difficult retrosternal gland can often be brought up into the neck by placing heavy silk sutures into the cervical component, applying progressive upward traction, placing more sutures successively as more and more of the gland comes into view.

This technique may not be appropriate for a soft friable gland and indeed it is essential at all times to avoid breaching the thyroid capsule for fear of seeding tumor in the case of an unsuspected malignant goiter. A preoperative diagnosis of malignancy is not possible in a retrosternal gland not amenable to palpation or fine needle aspiration for cytological evaluation. Significant bleeding difficult to control may also ensue if the thyroid capsule is breached before complete delivery of the gland.

It clearly follows that intracapsular fragmentation of the gland (morcellation) to facilitate gland removal should never be performed.

When a unilateral retrosternal component cannot be delivered into the neck in spite of performing all of the above maneuvers it may be helpful to remove the contralateral normal cervical lobe thus providing more space and surgical access in the neck.

If all of the above have led to an impasse and the goiter still remains in its retrosternal location a complete sternotomy and mediastinal approach will be required.

This technique, popularized by Dunhill ${ }^{7}$ and later by Taylor, ${ }^{8}$ is rarely necessary, but is required for extraordinarily large intrathoracic goiters where the thoracic inlet

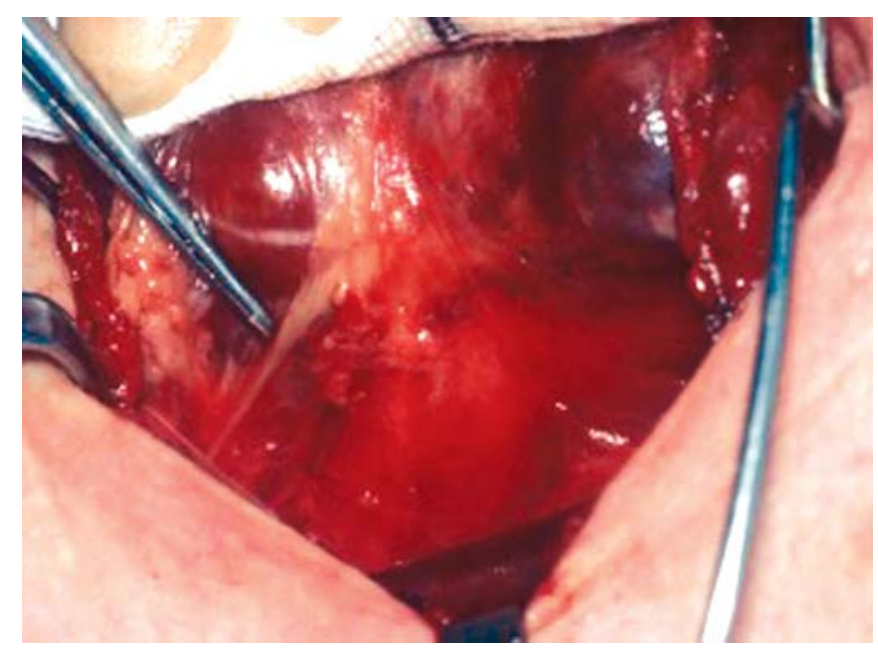

Fig. 1: Delivery of a recurrent retrosternal thyroid with the RLN seen to be displaced from its normal position and stretched over the surface of the nodular thyroid 
is simply to narrow for gland delivery, for tumors with malignant invasion and when excessive uncontrollable bleeding from deep in the chest is encountered. This latter situation is most likely to occur with a true ectopic intrathoracic thyroid deriving its principle blood supply from intrathoracic vessels. ${ }^{9}$ Fortunately, this is a rare occurrence.

The author has only encountered this situation on one occasion when successful delivery of a recurrent retrosternal goiter into the neck via a standard cervical approach was almost immediately followed by torrential hemorrhage from deep in the chest. After performing a sternotomy, the source of the bleeding was found to be a large arterial branch with an origin close to the root of the aorta.

A rare variety of retrosternal goiter which can pose a considerable surgical challenge is the thyroid which extends posteriorly from the left lobe crossing into the right posterior mediastinum behind the trachea and esophagus. Fortunately most glands of this type can still be delivered via a cervical approach. Rarely will it be necessary to add a right anterolateral thoracotomy through the fourth interspace to allow adequate exposure and safe dissection.

\section{SUMMARY}

Most retrosternal goiters can be safely resected through an entirely cervical approach with a low complication rate. On rare occasions a median sternotomy will be required for the thyroidectomy to be completed.

\section{REFERENCES}

1. Allo MD, Thompson NW. Rationale for the operative management of substernal goiters. Surgery 1983;94:969-77.

2. Sitges-Serra A, Sancho JJ. Surgical management of recurrent and intrathoracic goiters. In: Clark OH, Duh QY (Eds). Textbook of endocrine surgery. Philadelphia, Pennsylvania: WB Saunders 1997;262-74.

3. Katlic MR, Wang CC, Grillo HC. Substernal goiter. Ann Thorac Surg 1985;39:391-99.

4. Wheeler MH, Samuel MJ, Wade JSH. Management of retrosternal goiter. Br J Surg 1987;74:861.

5. Sanders LE, Rossi RL, Shahian DM, Williamson WA. Mediastinal goiters: The need for an aggressive surgical approach. Arch Surg 1992;127:609-13.

6. Michel LA, Bradpiece HA. Surgical management of substernal goiter. Br J Surg 1988;75:565-69.

7. Dunhill TP. Removal of intrathoracic tumors by the trans-sternal route. Br J Surg 1922;10:4-14.

8. Taylor S. Thyroid. In: Taylor S, Chisholm GD, O’Higgins N, Shields R (Eds). Surgical management. London: Heinemann 1984;513-24.

9. Wheeler MH. Clinical dilemma-retrosternal goitre. Br J Surg 1999;86:1235-36.

\section{ABOUT THE AUTHOR}

\section{Malcolm H Wheeler}

Formerly Professor, Department of Endocrine Surgery, University Hospital of Wales, Heath Park, Cardiff, CF4 4XW Wales United Kingdom

Correspondence Address: Aldbourne House, Cottrell Drive Bonvilston, Vale of Glamorgan, CF5 6TY, Wales, United Kingdom Phone: 0044(0)-1446-781126, e-mail: m.h.wheeler@btinternet.com 